Mathematical Modelling and Analysis

Volume 11 Number 2, 2006, PAGes 213-228

(c) 2006 Technika ISSN 1392-6292 print, ISSN 1648-3510 online

\title{
AN ANTIPLANE CONTACT PROBLEM FOR VISCOELASTIC MATERIALS WITH LONG-TERM MEMORY ${ }^{1}$
}

\author{
M. SOFONEA ${ }^{1}$, C. P. NICULESCU ${ }^{2}$ and A. MATEI ${ }^{2}$ \\ ${ }^{1}$ Laboratoire de Mathématiques et Physique pour les Systèmes, Université de \\ Perpignan \\ 52 Avenue Paul Alduy, F-66860 Perpignan, France \\ E-mail: sofonea@univ-perp.fr \\ ${ }^{2}$ Department of Mathematics, University of Craiova \\ A.I. Cuza Street 13, Craiova, RO-200585, Romania \\ E-mail: cniculescu@central.ucv.ro; andaluziamatei@k.ro \\ Received September 1, 2005; revised March 30, 2006; published online May 25, 2006
}

\begin{abstract}
We study a mechanical problem modeling the antiplane shear deformation of a cylinder in frictional contact with a rigid foundation. The material is assumed to be viscoelastic with long-term memory, the process is quasistatic, and the friction is modeled with Tresca's law. The problem leads to an evolutionary variational inequality that we study in an abstract framework. We then use the abstract result to prove the existence of a unique weak solution to the mechanical model. Moreover, we study its behavior with respect to the memory term and establish a convergence result.
\end{abstract}

Key words: antiplane problem, variational inequality, viscoelasticity with longterm memory, Tresca's friction law, approach to elasticity

\section{Introduction}

Nowadays a considerable progress has been achieved in the theory of variational inequalities and most of it was motivated by applications; its abstract character allows to solve wide classes of problems which present a common feature and to provide significant applications in the study of problems arising in sciences and engineering. The cross fertilization between modeling and applications on the one hand and nonlinear mathematical analysis on the other

\footnotetext{
${ }^{1}$ The work of the last two authors was performed in the framework of the Grant CNSIS 80/2005.
} 
hand was an important aspect which contributed to its development in the last four decades. Currently, the theory of variational inequalities became a fully mature discipline which deals with existence, uniqueness or nonuniqueness, regularity and continuous dependence results, together with numerical approximations and optimal control of the solutions. It provides results which are of considerable theoretical and applied interest.

The aim of this paper is to recall the attention to the great potential of inequalities in mechanics and physics. In the spirit of the classical book of G. Duvaut and J. L. Lions [3], we show how a concrete viscoelastic contact problem leads to a mathematical model which can be solved by using the methods of the theory of variational inequalities.

The contact between a deformable body and a foundation is a very frequent and important phenomenon. For this reason the literature covering this phenomenon is extensive, both in applied mathematics and in engineering or geophysics. The mathematical theory of contact mechanics is concerned with the mathematical structures which underline general problems of contact with different constitutive laws and different contact conditions, see for instance $[4,12]$ and the references therein. Its aim is to provide a sound, clear and rigorous background to the specific applied problems. A number of recent publications is dedicated to the study of quasistatic contact models involving viscoelastic materials with long-term memory. The variational analysis of such problems leads to evolutionary variational inequalities with integral term or with integro-differential term, see for instance $[9,10,11]$ for details.

In this paper we study the frictional contact between a deformable cylinder and a rigid foundation. We consider the case of antiplane shear deformation when the displacement is parallel to the generators of the cylinder and is independent of the axial coordinate. Such kind of problems were studied in a number of papers, in the context of various constitutive laws and contact conditions, see, e.g. $[1,5,8]$ and the references therein. The novelty in this paper consists in the fact that we model the friction with Tresca's law and the material's behavior with a viscoelastic constitutive law with long-term memory. We neglect the inertial term in the equation of motion to obtain a quasistatic approximation of the process. In the variational formulation, this mechanical problem leads to an integro-differential variational inequality. The main result we provide concerns the existence of a unique weak solution to the model. The proof is carried out in several steps, and it is based on arguments of evolutionary variational inequalities and Banach's fixed point theorem.

Our paper is structured as follows. In Section 2 we present the mechanical problem of antiplane contact, derive its variational formulation and state the main existence and uniqueness result, Theorem 1. In Section 3 we prove an abstract existence and uniqueness result, Theorem 2, and apply it in the proof of Theorem 1. Finally, in Section 4 we study the behavior of the solution as the memory term converges to zero and provide a convergence result, Theorem 4 . 


\section{The Antiplane Contact Problem and its Variational Formulation}

We consider a body $\mathcal{B}$ identified with a region in $\mathbb{R}^{3}$ it occupies in a fixed and undistorted reference configuration. We assume that $\mathcal{B}$ is a cylinder with generators parallel to the $x_{3}$-axes and a cross-section of $\mathcal{B}$ is a regular region $\Omega$ in the $x_{1}, x_{2}$-plane, $O x_{1} x_{2} x_{3}$ being a Cartesian coordinate system. The cylinder is assumed to be sufficiently long so that the end effects in the axial direction are negligible. Thus, $\mathcal{B}=\Omega \times(-\infty,+\infty)$. Let $\partial \Omega=\Gamma$. We assume that $\Gamma$ is divided into three disjoint measurable parts $\Gamma_{1}, \Gamma_{2}$ and $\Gamma_{3}$ such that the one-dimensional measure of $\Gamma_{1}$, denoted by meas $\Gamma_{1}$, is strictly positive. Let $T>0$ and let $[0, T]$ denote the time interval. The cylinder is clamped on $\Gamma_{1} \times(-\infty,+\infty)$ and is in contact with a rigid foundation on $\Gamma_{3} \times(-\infty,+\infty)$ during the process. Moreover, the cylinder is subjected to time dependent volume forces of density $\boldsymbol{f}_{0}$ on $\mathcal{B}$ and to time dependent surface tractions of density $\boldsymbol{f}_{2}$ on $\Gamma_{2} \times(-\infty,+\infty)$. We assume that

$$
\begin{aligned}
& \boldsymbol{f}_{0}=\left(0,0, f_{0}\right) \text { with } f_{0}=f_{0}\left(x_{1}, x_{2}, t\right): \Omega \times[0, T] \rightarrow \mathbb{R}, \\
& \boldsymbol{f}_{2}=\left(0,0, f_{2}\right) \text { with } f_{2}=f_{2}\left(x_{1}, x_{2}, t\right): \Gamma_{2} \times[0, T] \rightarrow \mathbb{R} .
\end{aligned}
$$

The body forces (2.1) and the surface tractions (2.2) would be expected to give rise to a deformation of the cylinder whose displacement, denoted by $\boldsymbol{u}$, is independent on $x_{3}$ and has the form

$$
\boldsymbol{u}=(0,0, u) \text { with } u=u\left(x_{1}, x_{2}, t\right): \Omega \times[0, T] \rightarrow \mathbb{R} .
$$

Such kind of deformation is called an antiplane shear, see for instance $[6,7]$ for details.

The infinitesimal strain tensor is denoted by $\varepsilon(\boldsymbol{u})=\left(\varepsilon_{i j}(\boldsymbol{u})\right)$ and the stress field by $\sigma=\left(\sigma_{i j}\right)$. Here and below, in order to simplify the notation, we do not indicate the dependence of various functions on $x_{1}, x_{2}$ or $t$.

The material is modeled by the following viscoelastic constitutive law with long-term memory,

$$
\boldsymbol{\sigma}=\lambda(\operatorname{tr} \boldsymbol{\varepsilon}(\boldsymbol{u})) \boldsymbol{I}+2 \mu \boldsymbol{\varepsilon}(\boldsymbol{u})+2 \int_{0}^{t} \theta(t-s) \boldsymbol{\varepsilon}(\boldsymbol{u}(s)) d s,
$$

where $\lambda>0$ and $\mu>0$ are the Lamé coefficients, $\operatorname{tr} \varepsilon(\boldsymbol{u})=\sum_{i=1}^{3} \varepsilon_{i i}(\boldsymbol{u}), \boldsymbol{I}$ is the unit tensor in $\mathbb{R}^{3}$ and $\theta:[0, T] \rightarrow \mathbb{R}$ is the relaxation function. In the antiplane context (2.3), keeping in mind (2.4), the stress field becomes

$$
\boldsymbol{\sigma}=\left(\begin{array}{ccc}
0 & 0 & \boldsymbol{\sigma}_{13} \\
0 & 0 & \boldsymbol{\sigma}_{23} \\
\boldsymbol{\sigma}_{31} & \boldsymbol{\sigma}_{32} & 0
\end{array}\right),
$$


where

$$
\begin{gathered}
\boldsymbol{\sigma}_{13}=\boldsymbol{\sigma}_{31}=\mu \partial_{x_{1}} u+\int_{0}^{t} \theta(t-s) \partial_{x_{1}} u(s) d s, \\
\boldsymbol{\sigma}_{23}=\sigma_{32}=\mu \partial_{x_{2}} u+\int_{0}^{t} \theta(t-s) \partial_{x_{2}} u(s) d s .
\end{gathered}
$$

Neglecting the inertial term in the equation of motion we obtain the quasistatic approximation for the process. Thus, taking into account (2.5), (2.1) and the previous equalities, the equation of equilibrium reduces to the scalar equation

$$
\mu \Delta u+\int_{0}^{t} \theta(t-s) \Delta u(s) d s+f_{0}=0 \quad \text { in } \quad \Omega \times(0, T) .
$$

As the cylinder is clamped on $\Gamma_{1} \times(-\infty,+\infty) \times(0, T)$, the displacement field vanishes there. Thus, (2.3) implies that

$$
u=0 \quad \text { on } \quad \Gamma_{1} \times(0, T) .
$$

Let $\nu$ denote the unit normal on $\Gamma \times(-\infty,+\infty)$. We have

$$
\boldsymbol{\nu}=\left(\nu_{1}, \nu_{2}, 0\right) \text { with } \nu_{i}=\nu_{i}\left(x_{1}, x_{2}\right): \Gamma \rightarrow \mathbb{R}, i=1,2 .
$$

For a vector $\boldsymbol{v}$ we denote by $v_{\nu}$ and $\boldsymbol{v}_{\tau}$ its normal and tangential components on the boundary, given by

$$
v_{\nu}=\boldsymbol{v} \cdot \boldsymbol{\nu}, \quad \boldsymbol{v}_{\tau}=\boldsymbol{v}-v_{\nu} \boldsymbol{\nu}
$$

In (2.7) and everywhere in this paper ". " represents the inner product on the space $\mathbb{R}^{d}(d=2$ or 3$)$. Moreover, throughout this paper the notation $|\cdot|$ will designate the Euclidean norm on $\mathbb{R}^{d}$, and a dot above a function will represent its derivative with respect to the time variable. For a given stress field $\boldsymbol{\sigma}$ we denote by $\sigma_{\nu}$ and $\boldsymbol{\sigma}_{\tau}$ the normal and the tangential components on the boundary, that is

$$
\sigma_{\nu}=(\boldsymbol{\sigma} \boldsymbol{\nu}) \cdot \boldsymbol{\nu}, \quad \boldsymbol{\sigma}_{\tau}=\boldsymbol{\sigma} \boldsymbol{\nu}-\sigma_{\nu} \boldsymbol{\nu}
$$

From (2.5) and (2.6) we deduce that the Cauchy stress vector is given by

$$
\boldsymbol{\sigma} \boldsymbol{\nu}=\left(0,0, \mu \partial_{\nu} u+\int_{0}^{t} \theta(t-s) \partial_{\nu} u(s) d s\right)
$$

Here and below we use the notation $\partial_{\nu} u=\partial_{x_{1}} u \nu_{1}+\partial_{x_{2}} u \nu_{2}$. Taking into account the traction boundary condition $\boldsymbol{\sigma} \boldsymbol{\nu}=\boldsymbol{f}_{2}$ on $\Gamma_{2} \times(0, T)$, it follows from (2.2) and (2.9) that

$$
\mu \partial_{\nu} u+\int_{0}^{t} \theta(t-s) \partial_{\nu} u(s) d s=f_{2} \quad \text { on } \quad \Gamma_{2} \times(0, T) .
$$


We now describe the contact condition on $\Gamma_{3} \times(-\infty,+\infty)$. First, from (2.3) and (2.6) we infer that $u_{\nu}=0$, which shows that the contact is bilateral, that is, the contact is kept during all the process. Using now (2.3), (2.6)-(2.8) we conclude that

$$
\boldsymbol{u}_{\tau}=(0,0, u), \quad \boldsymbol{\sigma}_{\tau}=\left(0,0, \mu \partial_{\nu} u+\int_{0}^{t} \theta(t-s) \partial_{\nu} u(s) d s\right)
$$

We assume that the friction is invariant with respect to the $x_{3}$ axis and is modeled with Tresca's friction law, that is the following estimates are valid on $\Gamma_{3} \times(0, T)$ :

$$
\left\{\begin{array}{l}
\left|\boldsymbol{\sigma}_{\tau}\right| \leq g \\
\left|\boldsymbol{\sigma}_{\tau}\right|<g \quad \Rightarrow \quad \dot{\boldsymbol{u}}_{\tau}=0, \\
\left|\boldsymbol{\sigma}_{\tau}\right|=g \quad \Rightarrow \quad \exists \beta \geq 0 \text { such that } \boldsymbol{\sigma}_{\tau}=-\beta \dot{\boldsymbol{u}}_{\tau} .
\end{array}\right.
$$

Here $g: \Gamma_{3} \rightarrow \mathbb{R}_{+}$is a given function, the friction bound, and $\dot{\boldsymbol{u}}_{\tau}$ represents the tangential velocity on the contact boundary. The strict inequality holds in the stick zone and the equality in the slip zone. Using now (2.10) it is straightforward to see that conditions (2.11) imply on $\Gamma_{3} \times(0, T)$

$$
\left\{\begin{array}{l}
\left|\mu \partial_{\nu} u+\int_{0}^{t} \theta(t-s) \partial_{\nu} u(s) d s\right| \leq g \\
\left|\mu \partial_{\nu} u+\int_{0}^{t} \theta(t-s) \partial_{\nu} u(s) d s\right|<g \Rightarrow \dot{u}=0 \\
\left|\mu \partial_{\nu} u+\int_{0}^{t} \theta(t-s) \partial_{\nu} u(s) d s\right|=g \Rightarrow \exists \beta \geq 0 \text { such that } \\
\mu \partial_{\nu} u+\int_{0}^{t} \theta(t-s) \partial_{\nu} u(s) d s=-\beta \dot{u} .
\end{array}\right.
$$

Finally, we prescribe the initial displacement,

$$
u(0)=u_{0} \text { in } \Omega,
$$

where $u_{0}$ is a given function on $\Omega$.

We collect the above equations and conditions to obtain the classical formulation of the antiplane problem for viscoelastic materials with long-term memory, in frictional contact with a foundation.

Problem $\mathcal{P}$. Find the displacement field $u: \Omega \times[0, T] \rightarrow \mathbb{R}$ such that

$$
\begin{array}{rlrl}
\mu \Delta u+\int_{0}^{t} \theta(t-s) \Delta u(s) d s+f_{0}=0 & \text { in } \Omega \times(0, T), \\
u=0 & \text { on } \Gamma_{1} \times(0, T), \\
\mu \partial_{\nu} u+\int_{0}^{t} \theta(t-s) \partial_{\nu} u(s) d s=f_{2} & & \text { on } \Gamma_{2} \times(0, T),
\end{array}
$$




$$
\left\{\begin{array}{l}
\left|\mu \partial_{\nu} u+\int_{0}^{t} \theta(t-s) \partial_{\nu} u(s) d s\right| \leq g, \\
\left|\mu \partial_{\nu} u+\int_{0}^{t} \theta(t-s) \partial_{\nu} u(s) d s\right|<g \Rightarrow \dot{u}=0, \\
\left|\mu \partial_{\nu} u+\int_{0}^{t} \theta(t-s) \partial_{\nu} u(s) d s\right|=g \Rightarrow \exists \beta \geq 0 \text { such that } \\
\mu \partial_{\nu} u+\int_{0}^{t} \theta(t-s) \partial_{\nu} u(s) d s=-\beta \dot{u} \\
u(0)=u_{0} \quad \text { in } \Omega .
\end{array}\right.
$$

Notice that once the displacement field $u$ which solves Problem $\mathcal{P}$ is known, then the stress tensor can be calculated using (2.4).

We derive now the variational formulation of the Problem $\mathcal{P}$. To this end we introduce the functional space

$$
V=\left\{v \in H^{1}(\Omega) \mid v=0 \text { on } \Gamma_{1}\right\} .
$$

Here and below we write $v$ for the trace $\gamma v$ of $v$ on $\Gamma$. Since meas $\Gamma_{1}>0$, the Friedrichs-Poincare inequality holds, that there exists a positive constant $C_{P}$ (which depends only on $\Omega$ and $\Gamma_{1}$ ) such that

$$
\|u\|_{H^{1}(\Omega)} \leq C_{P}\|\nabla u\|_{L^{2}(\Omega)^{2}} \quad \forall u \in V .
$$

We consider on $V$ the inner product given by

$$
(u, v)_{V}=\int_{\Omega} \nabla u \cdot \nabla v d x \quad \forall u, v \in V,
$$

and let $\|\cdot\|_{V}$ be the associated norm, i.e.

$$
\|v\|_{V}=\|\nabla v\|_{L^{2}(\Omega)^{2}} \quad \forall v \in V .
$$

Using (2.12), it follows that $\|\cdot\|_{H^{1}(\Omega)}$ and $\|\cdot\|_{V}$ are equivalent norms on $V$ and therefore $\left(V,\|\cdot\|_{V}\right)$ is a real Hilbert space. By Sobolev's trace theorem we deduce that there exists $C_{0}>0$ (depending only on $\Omega, \Gamma_{1}$ and $\Gamma_{3}$ ) such that

$$
\|v\|_{L^{2}\left(\Gamma_{3}\right)} \leq C_{0}\|v\|_{V} \quad \forall v \in V .
$$

If $\left(X,\|\cdot\|_{X}\right)$ represents a real Banach space, we denote by $C([0, T] ; X)$ the space of continuous functions from $[0, T]$ to $X$, with the norm

$$
\|x\|_{C([0, T] ; X)}=\max _{t \in[0, T]}\|x(t)\|_{X}
$$

and we use standard notations for the Lebesgue space $L^{2}(0, T ; X)$ as well as for the Sobolev space $W^{1,2}(0, T ; X)$. In particular, recall that the norm on the space $L^{2}(0, T ; X)$ is given by

$$
\|u\|_{L^{2}(0, T ; X)}=\left(\int_{0}^{T}\|u(t)\|_{X}^{2} d t\right)^{\frac{1}{2}}
$$


and the norm on the space $W^{1,2}(0, T ; X)$ is given by

$$
\|u\|_{W^{1,2}(0, T ; X)}=\left(\int_{0}^{T}\|u(t)\|_{X}^{2} d t+\int_{0}^{T}\|\dot{u}(t)\|_{X}^{2} d t\right)^{\frac{1}{2}} .
$$

Finally, we suppress the argument $X$ when $X=\mathbb{R}$; thus, for example, we use the notation $W^{1,2}(0, T)$ for the space $W^{1,2}(0, T ; \mathbb{R})$ and the notation $\|\cdot\|_{W^{1,2}(0, T)}$ for the norm $\|\cdot\|_{W^{1,2}(0, T ; \mathbb{R})}$.

In the study of the mechanical Problem $\mathcal{P}$ we assume that the friction bound function $g$ satisfies

$$
g \in L^{\infty}\left(\Gamma_{3}\right) \text { and } g(x) \geq 0 \quad \text { a.e. } x \in \Gamma_{3} .
$$

The forces and tractions are assumed to have the regularity

$$
f_{0} \in W^{1,2}\left(0, T ; L^{2}(\Omega)\right), \quad f_{2} \in W^{1,2}\left(0, T ; L^{2}\left(\Gamma_{2}\right)\right),
$$

and, for the relaxation function, we assume

$$
\theta \in W^{1,2}(0, T) .
$$

We consider the functional $j: V \rightarrow \mathbb{R}_{+}$given by

$$
j(v)=\int_{\Gamma_{3}} g|v| d a \quad \forall v \in V
$$

and let $f:[0, T] \rightarrow V$ be defined by

$$
(f(t), v)_{V}=\int_{\Omega} f_{0}(t) v d x+\int_{\Gamma_{2}} f_{2}(t) v d a \quad \forall v \in V, \quad t \in[0, T] .
$$

The definition of $f$ is based on Riesz's representation theorem and, by (2.15) and (2.18), we infer that

$$
f \in W^{1,2}(0, T ; V) .
$$

Finally, we assume that the initial data verifies

$$
u_{0} \in V
$$

and, moreover,

$$
\mu\left(u_{0}, v\right)_{V}+j(v) \geq(f(0), v)_{V} \quad \forall v \in V .
$$

Condition (2.21) represents a compatibility condition on the initial data that is necessary in many quasistatic problems, see for instance [12]. Physically, it is needed so as to guarantee that initially the state is in equilibrium, since otherwise the inertial terms cannot be neglected and the problem becomes dynamic.

Using Green's formula it is straightforward to derive the following variational formulation of Problem $\mathcal{P}$. 
Problem $\mathcal{P}_{V}$. Find a displacement field $u:[0, T] \rightarrow V$ such that

$$
\begin{aligned}
& \mu(u(t), v-\dot{u}(t))_{V}+\left(\int_{0}^{t} \theta(t-s) u(s) d s, v-\dot{u}(t)\right)_{V}+j(v) \\
& \quad-j(\dot{u}(t)) \geq(f(t), v-\dot{u}(t))_{V} \quad \forall v \in V, \quad \text { a.e. } t \in(0, T), \\
& u(0)=u_{0} .
\end{aligned}
$$

Our main existence and uniqueness result, which we establish in Section 3 , is the following.

Theorem 1. Assume that (2.14)-(2.16), (2.20) and (2.21) hold. Then there exists a unique solution of problem $\mathcal{P}_{V}$. Moreover, the solution satisfies $u \in$ $W^{1,2}(0, T ; V)$.

An element $u$ which solves Problem $\mathcal{P}_{V}$ is called a weak solution of the mechanical problem $\mathcal{P}$. We conclude by Theorem 1 that the antiplane contact problem $\mathcal{P}$ has a unique weak solution, provided that (2.14)-(2.16), (2.20) and (2.21) hold.

\section{An Abstract Existence and Uniqueness Result}

The following abstract problem includes Problem $\mathcal{P}_{V}$ as a special case.

Problem $\mathcal{P}_{V}^{A}$. Find $u:[0, T] \rightarrow X$ such that

$$
\begin{aligned}
& a(u(t), v-\dot{u}(t))+\left(\int_{0}^{t} \theta(t-s) u(s) d s, v-\dot{u}(t)\right)_{X}+j(v)-j(\dot{u}(t)) \\
& \quad \geq(f(t), v-\dot{u}(t))_{X} \quad \forall v \in X, \quad \text { a.e. } t \in(0, T) \\
& u(0)=u_{0} .
\end{aligned}
$$

Here $X$ is a real Hilbert space endowed with the inner product $(\cdot, \cdot)_{X}$ and the data $a, \theta, j, f, u_{0}$ will be described below. We denote by $\|\cdot\|_{X}$ the associated norm in $X$ and by $0_{X}$ the zero element of $X$.

We assume that:

$$
\begin{aligned}
& \left\{\begin{array}{l}
a: X \times X \rightarrow R \text { is a bilinear symmetric form and } \\
\text { (a) there exists } m>0 \text { such that } \\
|a(u, v)| \leq m\|u\|_{X}\|v\|_{X} \forall u, v \in X, \\
\text { (b) there exists } \alpha>0 \text { such that } a(u, u) \geq \alpha\|u\|_{X}^{2} \forall u \in X,
\end{array}\right. \\
& \qquad \theta \in W^{1,2}(0, T),
\end{aligned}
$$




$$
\begin{aligned}
& f \in W^{1,2}(0, T ; X), \\
& u_{0} \in X, \\
& a\left(u_{0}, v\right)+j(v) \geq(f(0), v)_{X} \quad \forall v \in X .
\end{aligned}
$$

We have the following existence and uniqueness result.

Theorem 2. Assume that (3.3)-(3.8) hold. Then, there exists a unique solution $u \in W^{1,2}(0, T ; X)$ to the problem $\mathcal{P}_{V}^{A}$.

We turn to the proof of Theorem 2 which will be carried out in several steps. To this end, in the rest of this section we assume that (3.3)-(3.8) hold and we recall the following result.

Theorem 3. ([2], p. 117). Let $\left(X,(\cdot, \cdot)_{X}\right)$ be a real Hilbert space and let $j$ : $X \rightarrow(-\infty,+\infty]$ be a convex lower semicontinuous functional. Assume that $j \not \equiv+\infty$, that is

$$
D(j)=\{v \in X \mid j(v)<\infty\} \neq \emptyset .
$$

Let $f \in W^{1,2}(0, T ; X)$ and $u_{0} \in X$ be such that

$$
\sup _{v \in D(j)}\left\{(f(0), v)_{X}-\left(u_{0}, v\right)_{X}-j(v)\right\}<+\infty .
$$

Then, there exists a unique element $u \in W^{1,2}(0, T ; X)$ which satisfies $u(0)=$ $u_{0}$ and

$$
(u(t), v-\dot{u}(t))_{X}+j(v)-j(\dot{u}(t)) \geq(f(t), v-\dot{u}(t))_{X},
$$

for all $v \in X$, and a.e. $t \in(0, T)$.

In the first step of the proof we introduce the set

$$
\mathcal{W}=\left\{\eta \in W^{1,2}(0, T ; X) \mid \eta(0)=0_{X}\right\}
$$

and we prove the following existence and uniqueness result.

Lemma 1. For all $\eta \in \mathcal{W}$ there exists a unique element $u_{\eta} \in W^{1,2}(0, T ; X)$ such that

$$
\begin{gathered}
a\left(u_{\eta}(t), v-\dot{u}_{\eta}(t)\right)+\left(\eta(t), v-\dot{u}_{\eta}(t)\right)_{X}+j(v)-j\left(\dot{u}_{\eta}(t)\right) \\
\geq\left(f(t), v-\dot{u}_{\eta}(t)\right)_{X} \quad \forall v \in X, \quad \text { a.e. } t \in(0, T), \\
u_{\eta}(0)=u_{0} .
\end{gathered}
$$

Proof. Let

$$
(u, v)_{a}=a(u, v) \quad \forall u, v \in X .
$$

It follows that $(\cdot, \cdot)_{a}$ is an inner product on the space $X$ and the associated norm, denoted $\|\cdot\|_{a}$, is equivalent to the norm $\|\cdot\|_{X}$ on $X$. Consequently, $\left(X,(\cdot, \cdot)_{a}\right)$ is a real Hilbert space.

Let $f_{\eta}:[0, T] \rightarrow X$ be the function defined by 


$$
\left(f_{\eta}(t), v\right)_{a}=(f(t), v)_{X}-(\eta(t), v)_{X} \quad \forall v \in X, t \in[0, T] .
$$

Then, (3.6) and the regularity $\eta \in \mathcal{W}$ imply that

$$
f_{\eta} \in W^{1,2}(0, T ; X) .
$$

Moreover, using (3.9), (3.12) and (3.13), we obtain

$$
\left(f_{\eta}(0), v\right)_{a}-\left(u_{0}, v\right)_{a}-j(v)=(f(0), v)_{X}-a\left(u_{0}, v\right)-j(v) \quad \forall v \in X
$$

and, taking into account (3.8), we find

$$
\sup _{v \in D(j)}\left\{\left(f_{\eta}(0), v\right)_{A}-\left(u_{0}, v\right)_{A}-j(v)\right\}<+\infty .
$$

Keeping in mind (3.5), (3.7), (3.14) and (3.15), we can use Theorem 3 on the space $\left(X,(\cdot, \cdot)_{a}\right)$ to obtain the existence of a unique element $u_{\eta} \in$ $W^{1,2}(0, T ; X)$ such that $u_{\eta}(0)=u_{0}$ and

$\left(u_{\eta}(t), v-\dot{u}_{\eta}(t)\right)_{a}+j(v)-j\left(\dot{u}_{\eta}(t)\right) \geq\left(f_{\eta}(t), v-\dot{u}_{\eta}(t)\right)_{a} \forall v \in X$, a.e. $t \in(0, T)$.

It follows from (3.12) and (3.13) that $u_{\eta}$ is the unique solution to the problem (3.10)-(3.11) which concludes the proof.

In the next step we consider the operator $\Lambda: \mathcal{W} \rightarrow \mathcal{W}$ defined by

$$
\Lambda \eta(t)=\int_{0}^{t} \theta(t-s) u_{\eta}(s) d s, \quad \forall \eta \in \mathcal{W}, t \in[0, T] .
$$

It follows from (3.4) that the operator $\Lambda$ is well defined, since $\eta \in \mathcal{W}$ implies $\Lambda \eta \in \mathcal{W}$. We also note that

$$
\left(\frac{d}{d t} \Lambda \eta\right)(t)=\theta(0) u_{\eta}(t)+\int_{0}^{t} \dot{\theta}(t-s) u_{\eta}(s) d s \quad \forall \eta \in \mathcal{W}, \quad \text { a.e. } t \in(0, T) .
$$

We have the following result.

Lemma 2. The operator $\Lambda$ has a unique fixed point $\eta^{*} \in \mathcal{W}$.

Proof. Let $\eta_{1}, \eta_{2} \in \mathcal{W}$ and, for the sake of simplicity, denote $u_{1}=u_{\eta_{1}}$ and $u_{2}=u_{\eta_{2}}$. Using (3.16) and (3.4) it follows that

$$
\left\|\Lambda \eta_{1}(t)-\Lambda \eta_{2}(t)\right\|_{X}^{2} \leq c \int_{0}^{t}\left\|u_{1}(s)-u_{2}(s)\right\|_{X}^{2} d s \quad \forall t \in[0, T] .
$$

Here and in what follows $c$ represents a generic positive constant which may depend on $\|\theta\|_{W^{1,2}(0, T)}, a$ and $T$, and whose value may change from place to place.

Moreover, from (3.17) we infer that 


$$
\begin{aligned}
& \left\|\left(\frac{d}{d t} \Lambda \eta_{1}\right)(t)-\left(\frac{d}{d t} \Lambda \eta_{2}\right)(t)\right\|_{X} \\
& \quad \leq|\theta(0)|\left\|u_{1}(t)-u_{2}(t)\right\|_{X}+\int_{0}^{t}|\dot{\theta}(t-s)|\left\|u_{1}(s)-u_{2}(s)\right\|_{X} d s \\
& \quad \leq c\left(\left\|u_{1}(t)-u_{2}(t)\right\|_{X}+\left(\int_{0}^{t}\left\|u_{1}(s)-u_{2}(s)\right\|_{X}^{2} d s\right)^{\frac{1}{2}}\right) \quad \text { a.e. } t \in(0, T),
\end{aligned}
$$

which yields

$$
\begin{aligned}
& \left\|\left(\frac{d}{d t} \Lambda \eta_{1}\right)(t)-\left(\frac{d}{d t} \Lambda \eta_{2}\right)(t)\right\|_{X}^{2} \\
& \quad \leq c\left(\left\|u_{1}(t)-u_{2}(t)\right\|_{X}^{2}+\int_{0}^{t}\left\|u_{1}(s)-u_{2}(s)\right\|_{X}^{2} d s\right) \quad \text { a.e. } t \in(0, T) .
\end{aligned}
$$

On the other hand, taking into account (3.10), we have the inequalities

$$
\begin{aligned}
a\left(u_{1}(s), v\right. & \left.-\dot{u}_{1}(s)\right)+\left(\eta_{1}(s), v-\dot{u}_{1}(s)\right)_{X}+j(v)-j\left(\dot{u}_{1}(s)\right) \\
\geq & \left(f(s), v-\dot{u}_{1}(s)\right)_{X}, \\
a\left(u_{2}(s), v\right. & \left.-\dot{u}_{2}(s)\right)+\left(\eta_{2}(s), v-\dot{u}_{2}(s)\right)_{X}+j(v)-j\left(\dot{u}_{2}(s)\right) \\
\geq & \left(f(s), v-\dot{u}_{2}(s)\right)_{X},
\end{aligned}
$$

for all $v \in X$, a.e. $s \in(0, T)$. We choose $v=\dot{u}_{2}(s)$ in the first inequality, $v=\dot{u}_{1}(s)$ in the second inequality, add the results and use (3.12) to obtain

$$
\frac{1}{2} \frac{d}{d s}\left\|u_{1}(s)-u_{2}(s)\right\|_{a}^{2} \leq-\left(\eta_{1}(s)-\eta_{2}(s), \dot{u}_{1}(s)-\dot{u}_{2}(s)\right)_{X} \quad \text { a.e. } s \in(0, T) .
$$

Let $t \in[0, T]$. Integrating the previous inequality from 0 to $t$ and using (3.11), we get

$$
\begin{aligned}
\frac{1}{2}\left\|u_{1}(t)-u_{2}(t)\right\|_{a}^{2} & \leq-\left(\eta_{1}(t)-\eta_{2}(t), u_{1}(t)-u_{2}(t)\right)_{X} \\
& +\int_{0}^{t}\left(\dot{\eta}_{1}(s)-\dot{\eta}_{2}(s), u_{1}(s)-u_{2}(s)\right)_{X} d s .
\end{aligned}
$$

Now it follows that

$$
\begin{aligned}
c\left\|u_{1}(t)-u_{2}(t)\right\|_{X}^{2} & \leq\left\|\eta_{1}(t)-\eta_{2}(t)\right\|_{X}\left\|u_{1}(t)-u_{2}(t)\right\|_{X} \\
& +\int_{0}^{t}\left\|\dot{\eta}_{1}(s)-\dot{\eta}_{2}(s)\right\|\left\|_{X}\right\| u_{1}(s)-u_{2}(s) \|_{X} d s
\end{aligned}
$$

and, using the inequality

$$
a b \leq \frac{a^{2}}{2 \alpha}+2 \alpha b^{2}, \quad \text { for } a, b, \alpha>0,
$$

we find 


$$
\begin{aligned}
\left\|u_{1}(t)-u_{2}(t)\right\|_{X}^{2} & \leq c\left(\left\|\eta_{1}(t)-\eta_{2}(t)\right\|_{X}^{2}+\int_{0}^{t}\left\|\dot{\eta}_{1}(s)-\dot{\eta}_{2}(s)\right\|_{X}^{2} d s\right. \\
& \left.+\int_{0}^{t}\left\|u_{1}(s)-u_{2}(s)\right\|_{X}^{2} d s\right) .
\end{aligned}
$$

As

$$
\eta_{1}(t)-\eta_{2}(t)=\int_{0}^{t}\left(\dot{\eta}_{1}(s)-\dot{\eta}_{2}(s)\right) d s
$$

we deduce that

$$
\left\|\eta_{1}(t)-\eta_{2}(t)\right\|_{X}^{2} \leq c \int_{0}^{t}\left\|\dot{\eta}_{1}(s)-\dot{\eta}_{2}(s)\right\|_{X}^{2} d s .
$$

Plugging this inequality into (3.20) we obtain

$$
\left\|u_{1}(t)-u_{2}(t)\right\|_{X}^{2} \leq c\left(\int_{0}^{t}\left\|\dot{\eta}_{1}(s)-\dot{\eta}_{2}(s)\right\|_{X}^{2} d s+\int_{0}^{t}\left\|u_{1}(s)-u_{2}(s)\right\|_{X}^{2} d s\right) .
$$

Applying now Gronwall's inequality we deduce

$$
\left\|u_{1}(t)-u_{2}(t)\right\|_{X}^{2} \leq c \int_{0}^{t}\left\|\dot{\eta}_{1}(s)-\dot{\eta}_{2}(s)\right\|_{X}^{2} d s
$$

which yields

$$
\int_{0}^{t}\left\|u_{1}(s)-u_{2}(s)\right\|_{X}^{2} d s \leq c \int_{0}^{t}\left\|\dot{\eta}_{1}(s)-\dot{\eta}_{2}(s)\right\|_{X}^{2} d s .
$$

Combining now (3.18), (3.19), (3.21) and (3.22) we obtain

$$
\left\|\Lambda \eta_{1}(t)-\Lambda \eta_{2}(t)\right\|_{X}^{2}+\left\|\left(\frac{d}{d t} \Lambda \eta_{1}\right)(t)-\left(\frac{d}{d t} \Lambda \eta_{2}\right)(t)\right\|_{X}^{2} \leq c \int_{0}^{t}\left\|\dot{\eta}_{1}(s)-\dot{\eta}_{2}(s)\right\|_{X}^{2} d s .
$$

Iterating the last inequality $p$ times we infer

$$
\begin{aligned}
\| \Lambda^{p} \eta_{1}(t)-\Lambda^{p} \eta_{2}(t) & \left\|_{X}^{2}+\right\|\left(\frac{d}{d t} \Lambda^{p} \eta_{1}\right)(t)-\left(\frac{d}{d t} \Lambda^{p} \eta_{2}\right)(t) \|_{X}^{2} \\
& \leq c^{p} \int_{0}^{t} \int_{0}^{s_{1}} \cdots \int_{0}^{s_{p-1}}\left\|\dot{\eta}_{1}\left(s_{p}\right)-\dot{\eta}_{2}\left(s_{p}\right)\right\|_{X}^{2} d s_{p} \cdots d s_{1},
\end{aligned}
$$

where $\Lambda^{p}$ denotes the power of the operator $\Lambda$. The last inequality implies

$$
\left\|\Lambda^{p} \eta_{1}-\Lambda^{p} \eta_{2}\right\|_{W^{1,2}(0, T ; X)}^{2} \leq \frac{c^{p} T^{p}}{p !}\left\|\eta_{1}-\eta_{2}\right\|_{W^{1,2}(0, T ; X)}^{2} .
$$

Since $\lim _{p \rightarrow \infty} \frac{c^{p} T^{p}}{p !}=0$, the previous inequality implies that a power $\Lambda^{p}$ of $\Lambda$ is a contraction in $\mathcal{W}$ for $p$ large enough. It follows now from Banach's fixed point theorem that there exists a unique element $\eta^{*} \in \mathcal{W}$ such that $\Lambda^{p} \eta^{*}=\eta^{*}$. Moreover, since 


$$
\Lambda^{p}\left(\Lambda \eta^{*}\right)=\Lambda\left(\Lambda^{p} \eta^{*}\right)=\Lambda \eta^{*},
$$

we deduce that $\Lambda \eta^{*}$ is also a fixed point of the operator $\Lambda^{p}$. By the uniqueness of the fixed point, we conclude that $\Lambda \eta^{*}=\eta^{*}$, which shows that $\eta^{*}$ is a fixed point of $\Lambda$. The uniqueness of the fixed point of the operator $\Lambda$ follows from the uniqueness of the fixed point of the operator $\Lambda^{p}$.

We have now all the ingredients to prove the theorem.

Proof of Theorem 2. Existence. Let $\eta^{*} \in \mathcal{W}$ be the fixed point of $\Lambda$ and let $u_{\eta^{*}}$ be the function defined by Lemma 1 for $\eta=\eta^{*}$. Since $\Lambda \eta^{*}=\eta^{*}$, it follows from (3.16), (3.10) and (3.11) that $u_{\eta^{*}}$ is a solution to the problem (3.1)-(3.2). Moreover, the regularity $u_{\eta^{*}} \in W^{1,2}(0, T ; X)$ is obtained from Lemma 1 .

Uniqueness. The uniqueness follows by arguments similar to those used in [11] and is a consequence of the uniqueness of the fixed point of the operator $\Lambda$. Indeed, let $u$ be a solution of problem (3.1)-(3.2) with regularity $u \in$ $W^{1,2}(0, T ; X)$ and consider the element $\eta \in \mathcal{W}$ defined by

$$
\eta(t)=\int_{0}^{t} \theta(t-s) u(s) d s \quad \forall t \in[0, T] .
$$

Then $u$ is also the solution of problem (3.10)-(3.11), so by Lemma 1 we have

$$
u=u_{\eta}
$$

By (3.16), (3.23) and (3.24) we obtain that $\Lambda \eta=\eta$. As $\Lambda$ has a unique fixed point, we conclude that

$$
\eta=\eta^{*} \text {. }
$$

The uniqueness part of Theorem 2 is now a consequence of equalities (3.24) and (3.25).

We now apply Theorem 2 to provide the well-posedness of problem $\mathcal{P}_{V}$.

Proof of Theorem 1. Consider the form $a: V \times V \rightarrow \mathbb{R}$ defined by

$$
a(u, v)=\mu \int_{\Omega} \nabla u \cdot \nabla v d x \quad \forall u, v \in V .
$$

Clearly this form is bilinear, continuous, coercive and symmetric; moreover, using (2.13) and (2.14) it follows that the functional $j$ defined by (2.17) is convex, lower semicontinuous and proper. Therefore, we conclude that conditions (3.3) and (3.5) are satisfied in this case. Taking into account (2.16) and (2.19)-(2.21), the conclusion of Theorem 1 follows from Theorem 3.

\section{A Convergence Result}

In this section we investigate the behavior of the weak solution of the antiplane contact problem as the relaxation function converges to zero. To this 
end, in order to underline the dependence with respect to the function $\theta$, we reformulate the problem $\mathcal{P}_{V}$ as follows.

Problem $\mathcal{P}_{V}^{\theta}$. Find a displacement field $u_{\theta}:[0, T] \rightarrow V$ such that

$$
\begin{aligned}
& \mu\left(u_{\theta}(t), v-\dot{u}_{\theta}(t)\right)_{V}+\left(\int_{0}^{t} \theta(t-s) u_{\theta}(s) d s, v-\dot{u}_{\theta}(t)\right)_{V}+j(v) \\
& \quad-j\left(\dot{u}_{\theta}(t)\right) \geq\left(f(t), v-\dot{u}_{\theta}(t)\right)_{V} \quad \forall v \in V, \quad \text { a.e. } t \in(0, T), \\
& u_{\theta}(0)=u_{0} .
\end{aligned}
$$

We also consider the problem obtained for $\theta=0$ that is:

Problem $\mathcal{P}_{V}^{0}$. Find a displacement field $u:[0, T] \rightarrow V$ such that

$$
\begin{gathered}
\mu(u(t), v-\dot{u}(t))_{V}+j(v)-j(\dot{u}(t)) \geq(f(t), v-\dot{u}(t))_{V} \\
\forall v \in V, \quad \text { a.e. } t \in(0, T), \\
u(0)=u_{0} .
\end{gathered}
$$

Clearly problem $\mathcal{P}_{V}^{0}$ represents the variational formulation of the antiplane frictional contact problem for linear elastic materials, i.e. the problem obtained when (2.4) is replaced by the elastic constitutive law

$$
\sigma=\lambda(\operatorname{tr}(\varepsilon(\boldsymbol{u}))) \boldsymbol{I}+2 \mu \varepsilon(\boldsymbol{u}) .
$$

Everywhere in this section we assume that (2.14)-(2.16), (2.20) and (2.21) hold. It follows from Theorem 1 that Problem $\mathcal{P}_{V}^{\theta}$ has a unique solution $u_{\theta} \in$ $W^{1,2}(0, T ; V)$ and Problem $\mathcal{P}_{V}^{0}$ has a unique solution $u \in W^{1,2}(0, T ; V)$.

Consider now the assumption

$$
\|\theta\|_{W^{1,2}(0, T)} \rightarrow 0
$$

Our main result in this section is the following.

Theorem 4. Assume that (2.14)-(2.16), (2.20), (2.21) and (4.5) hold. Then the solution $u_{\theta}$ of the Problem $\mathcal{P}_{V}^{\theta}$ converge to the solution $u$ of the Problem $\mathcal{P}_{V}^{0}$, i.e.

$$
\left\|u_{\theta}-u\right\|_{C([0, T] ; V)} \rightarrow 0
$$

Proof. Denote

$$
\eta_{\theta}(t)=\int_{0}^{t} \theta(t-s) u_{\theta}(s) d s \quad \forall t \in[0, T] .
$$

Using (4.1), (4.3), (4.7) and arguments similar to those used to obtain (3.21), yields

$$
\left\|u_{\theta}(t)-u(t)\right\|_{V}^{2} \leq c \int_{0}^{t}\left\|\dot{\eta}_{\theta}(s)\right\|_{V}^{2} d s \quad \forall t \in[0, T] .
$$


Here and below $c$ represent a positive constant which may depend on $u$ but is independent of $\theta$ and whose value may change from line to line. It follows from (4.7) that

$$
\dot{\eta}_{\theta}(t)=\theta(0) u_{\theta}(t)+\int_{0}^{t} \dot{\theta}(t-s) u_{\theta}(s) d s, \quad \text { a.e. } t \in(0, T)
$$

which implies the inequality

$$
\begin{aligned}
& \left\|\dot{\eta}_{\theta}(t)\right\|_{V} \leq|\theta(0)|\left\|u_{\theta}(t)-u(t)\right\|_{V}+|\theta(0)|\|u(t)\|_{V}+\int_{0}^{t}|\dot{\theta}(t-s)| \\
& \times\left\|u_{\theta}(s)-u(s)\right\|_{V} d s+\int_{0}^{t}|\dot{\theta}(t-s)|\|u(s)\|_{V} d s \quad \text { a.e. } t \in(0, T) .
\end{aligned}
$$

Moreover (4.5) implies that there exists $c>0$ such that

$$
\|\theta\|_{C(0, T)} \leq c, \quad\|\dot{\theta}\|_{L^{2}(0, T)} \leq c .
$$

Keeping in mind (4.9) and (4.10), after some algebra yields

$$
\left\|\dot{\eta}_{\theta}(t)\right\|_{V}^{2} \leq c\left(\|\theta\|_{W^{1,2}(0, T)}^{2}+\left\|u_{\theta}(t)-u(t)\right\|_{V}^{2}+\int_{0}^{t}\left\|u_{\theta}(s)-u(s)\right\|_{V}^{2} d s\right)
$$

We integrate the previous inequality to find

$$
\int_{0}^{t}\left\|\dot{\eta}_{\theta}(s)\right\|_{V}^{2} d s \leq c\left(\|\theta\|_{W^{1,2}(0, T)}^{2}+\int_{0}^{t}\left\|u_{\theta}(s)-u(s)\right\|_{V}^{2} d s\right) \quad \forall t \in[0, T] .
$$

We combine (4.8) and (4.11) to obtain

$$
\left\|u_{\theta}(t)-u(t)\right\|_{V}^{2} \leq c\left(\|\theta\|_{W^{1,2}(0, T)}^{2}+\int_{0}^{t}\left\|u_{\theta}(s)-u(s)\right\|_{V}^{2} d s\right) \quad \forall t \in[0, T],
$$

and, using Gronwall's inequality, we find

$$
\left\|u_{\theta}(t)-u(t)\right\|_{V} \leq c \|\left.\theta\right|_{W^{1,2}(0, T)} \quad \forall t \in[0, T] .
$$

The convergence result (4.6) is now a consequence of (4.5) and (4.12).

From Theorem 4 we conclude that the weak solution to the antiplane viscoelastic problem with Tresca's friction law may be approached by the weak solution to the antiplane elastic problem with Tresca's friction law as the relaxation function is small enough. In addition to the mathematical interest in this result, this is of importance from mechanical point of view, as it indicates that the elasticity with friction may be considered as a limit case of viscoelasticity with friction as the memory decreases. 


\section{References}

[1] F. Andreu, J.M. Mazón and M. Sofonea. Entropy solutions in the study of antiplane shear deformations for elastic solids. Math. Models Methods Appl. Sci., 10, 96-126, 2000.

[2] H. Brézis. Problèmes unilatéraux. J. Math. Pures et Appl., 51, 1-168, 1972.

[3] G. Duvaut and J.L. Lions. Inequalities in Mechanics and Physics. SpringerVerlag, Berlin, 1976.

[4] W. Han and M. Sofonea. Quasistatic contact problems in viscoelasticity and viscoplasticity. Studies in Advanced Mathematics, American Mathematical Society, Providence, RI-International Press, Somerville, MA, 30, 2002.

[5] T.-V. Hoarau-Mantel and A. Matei. Analysis of a viscoelastic antiplane contact problem with slip-dependent friction. Int. J. Appl. Math. Comput. Sci., 12, 51$58,2002$.

[6] C.O. Horgan. Anti-plane shear deformation in linear and nonlinear solid mechanics. SIAM Rev., 37, 53-81, 1995.

[7] C.O. Horgan and K.L. Miller. Anti-plane shear deformation for homogeneous and inhomogeneous anisotropic linearly elastic solids. J. Appl. Mech., 61, 23$29,1994$.

[8] A. Matei, V.V. Motreanu and M. Sofonea. A quasistatic antiplane contact problem with slip dependent friction. Advances in Nonlinear Variational Inequalities, 4, 1-21, 2001.

[9] A. Rodríguez-Arós, M. Sofonea and J.M. Viano. Numerical analysis of a class of evolutionary variational inequalities with integral term. Ann. Univ. Craiova, Math-Info., 30, 30-37, 2003.

[10] A. Rodríguez-Arós, M. Sofonea and J.M. Viano. A signorini frictionless contact problem for viscoelastic materials with long-term memory. In: F. Brezzi, A. Buffo, S. Corsaro and A. Murli(Eds.), Numerical Mathematics and Advanced Applications-Enumath 2001 European Conference on Numerical Mathematics and Advances Applications, Springer-Verlag, Milano, 527-535, 2003.

[11] A. Rodríguez-Arós, M. Sofonea and J.M. Viano. A class of evolutionary variational inequalities with Volterra-type integral term. Math. Models Methods Appl. Sci., 14, 557-577, 2004.

[12] M. Shillor, M. Sofonea and J.J. Telega. Models and Variational Analysis of Quasistatic Contact, volume 655. Springer, Berlin Heidelberg, 2004. 